\title{
Efficient Routing in Mobile Adhoc Networks Emphasizing Quality of Service by Trust \& Energy based AODV
}

\author{
S. Sridhar, and R. Baskaran
}

\begin{abstract}
Attention in the region of Mobile Ad-hoc Network (MANET) is increasing because of its realistic applications and necessity of communication in mobile devices. A mobile ad hoc network consists of mobile self configuring wireless nodes and these nodes communicate between them without any centralized management. The dynamic characteristics of MANET, has made it fairly demanding to uphold connectivity and guarantee Quality of Service (QoS). The trust based routing is one way to form cooperation among nodes for performing an efficient routing between nodes. In this paper a trust and energy based AODV is presented where nodes are selected for routing based on its trust and energy value. Trust is calculated based on the nodes success and failure rate. Energy calculated based on consumed and remaining energy values. A threshold value is defined and nodes are preferred for routing only if its trust and energy levels are higher than threshold. The work is implemented and simulated on NS-2. The simulation results have shown improvement on QoS metrics when compared with traditional AODV and DSR.
\end{abstract}

Index Terms - AODV, DSR, MANET, QoS, Trust

\section{INTRODUCTION}

A MANET is a peer-to-peer wireless network where nodes can communicate with each other without the use of infrastructure. Nodes can join and leave the network at anytime and are free to move arbitrarily and organize themselves randomly. The simplicity of deployment and the infrastructure less feature of Mobile Ad hoc Networks makes it extremely attractive for the present day scenarios. The dynamic topology changes are caused by quick and random node mobility that makes routing tough in MANET. Traditional routing protocols in MANET assume a collaborating environment inside the network. However, this suggestion is not always true and an aggressive or vulnerable environment can seriously affect network performance. MANET is a cooperation based network that expects each participating node to forward packets. This nature of MANET leads to the possibility that there could be some malicious nodes that try negotiating the routing protocol functionality and makes MANET vulnerable.

Manuscript received February 7, 2015; revised April 12, 2015.

S. Sridhar is with the Head/Department of Computer Applications, S.A.Engineering College, Chennai - 77 (e-mail: sridhar@saec.ac.in).

R. Baskaran is with the Department of Computer Science and Engineering, CEG, Guindy, Anna University, Chennai - 25 (e-mail:

baskaran.ramachandran@gmail.com).
Routing protocols in MANETs are generally classified as proactive and reactive [1]. Reactive routing protocols start to establish routes only when required or only if there is a demand. These kinds of protocols are based on RREQ (Route Request) and RREP (Route Reply) messages. The purpose of RREQ message is to discover a route from source to destination node. When the destination node gets a RREQ message, it sends RREP message along the established path. There are many reactive routing protocols, such as ad hoc ondemand distance vector (AODV) [2], dynamic source routing (DSR) [3], temporally order routing algorithm (TORA)[4], associativity-based routing (ABR) [5], signal stability-based adaptive (SSA) [6] and relative distance microdiscovery ad hoc routing (RDMAR) [7]. The other classification of routing protocol is table-driven or pro-active routing protocols. Each node maintains one or more routing information table of all the participating nodes and updates their routing information regularly to maintain most up-to-date analysis of the network. Here there is no concept of RREQ or RREP and only route updates are exchanged as control messages among nodes to update their routing information. There are many proactive routing protocols, such as destination sequenced distance vector (DSDV) [8], wireless routing protocol (WRP) [9], clusterhead gateway switch routing (CGSR) [10] and fisheye state routing (FSR) [11].

Due to the vibrant nature of Mobile Ad-Hoc Networks, there are many issues which need to be dealt with and one of the areas for perfection is Quality of Service (QoS). When it comes to QoS routing, the routing protocols have to ensure that the QoS requirements are met [12]. A few challenges faced in providing Qos are steadily changing environment, unobstructed mobility which causes recurring path breaks and also make the link-specific and state-specific information in the nodes to be inexact.

The idea of using trust to mitigate security threats has been an important area of research [13]. The concept of "Trust" originally derives from social sciences and is defined as the degree of subjective belief about the behaviors of a particular entity [14]. The trust based routing is one way to form cooperation among nodes for establishing an efficient routing between nodes. Trust value plays a crucial role in all of the network activities. Continuous evaluation of node's performance is used to calculate the trust of this node. Basically Mobile ad hoc networks are designed for a cooperative environment but in hostile environments trustbased routing should be used. Instead of establishing the shortest routes as done in traditional routing protocols, 
trusted routes are established to make it a trustworthy routing. In addition to trust the energy values of nodes are also considered for routing. Energy plays a vital role in MANET routing. Nodes may drain out of energy based on the transmission and reception. Transmission power of nodes also affects this energy level directly. The additional overheads (calculation of trust and energy) will further drain the power resources of mobile nodes which are anyway likely to be running on limited battery power. Avoid nodes which have low energy [15]. If nodes which are highly trusted run out of energy then it is waste of putting in the overhead of calculating trust and then considering the node for routing.

Thus considering the above cases a trust and energy based AODV is proposed which would address the issues raised and also improves the Qos metrics. As per the proposed work the nodes which are to be selected for routing will be selected based on its trust and energy value. Trust value of a node is calculated based on its success and failure rate. A threshold is set which is the average trust value of the nodes which are the eligible for next hop node. The node which has the highest trust value than the threshold is selected for routing. The energy value of the node calculated based on its consumed and remaining energy. Therefore it is wise to select a node for efficient routing by considering its trust and energy values.

\section{LITERATURE SURVEY}

A reliable routing algorithm based on fuzzy logic [16] is proposed where three parameters are determined: trust value, energy value and reliability value which are used for finding a stable route from source to destination. During route discovery, every node records its trust value and energy capacity in RREQ packet .In the destination ,based on reliability value, is decided which route is selected .The path with more reliability value is permitted to route data packets from source to destination. The proposed method has significant reliability improvement in comparison with AODV.

Study on the reliability-oriented transmission service in WSNs is made. Three key problems are identified and investigated. To alleviate these problems, a novel in-middle recovery mechanism that fills the gap between the traditional local and E2E recovery mechanisms is proposed [17]. To realize the idea, proliferation routing scheme is designed and implemented. Proliferation routing has three independent components, each targeting at one specific problem. Carefully designed system parameters grant proliferation routing more flexibility and more freedom to an E2E transmission service quality. Analysis and experiment results showed that the success rate can be maintained up to 80 percent in an energyefficient way compared with the traditional approaches.

A modified AODV routing protocol called MAODV [18] is presented, which takes route stability into consideration to attempt to establish a more stable path between the source and destination. some changes in Hello and RREQ message format are made to respectively record the sending time and route stability factor. MAODV shows a better performance. The performance of reactive routing protocol is analyzed via increasing number of nodes and observing its effect on Quality of Service (QoS) of Mobile Adhoc Network
[19]. The QoS depends upon several parameters like end-end delay, throughput, data drop and network load. The reactive routing protocol considered here is AODV with MCHG. The performance of Routing Protocol is observed via enhancing the network size on the basis of following parameters: delay, throughput, traffic sent, traffic received, data dropped and network load. Simulation experiments conducted in the conditions where QoS of MANET is improved

AODV is enhanced to provide QoS, where route discovery process is modified to reduce the connection set up latency. In addition, QoS provision based on delay and bandwidth requirements is incorporated to ensure guaranteed performance level to the QoS sensitive applications. The enhanced protocol is called NQoS AODV [20]. Three performance parameters: End-to-end Average Delay, Packet Delivery Ratio and Control Overhead are considered in different mobility scenarios to compare the performance of the proposed protocol with the original AODV. This paper proposes and investigates a power-aware ad hoc on-demand distance vector routing protocol (PAW-AODV) for efficient power routing [21]. PAW-AODV could use the limited power resources efficiently as it routes based on a power-based cost function. Both AODV and PAW-AODV are simulated in various mobile situations and are also subjected to the various hop count limits that the data could traverse from source to destination. Their performances in the various scenarios are then compared to reflect the relative merits of each protocol.

The enhanced AODV routing protocol [22], modified to improve the networks lifetime in MANET. One improvement for the AODV protocol is to maximize the networks lifetime by applying an Energy Mean Value algorithm which considerate node energy-aware. The effectiveness of enhanced AODV compared with existing AODV. Two malicious attacks (gray, black hole) and two types of selfish behaviors (type-1, type-2) are considered [23]. The objective of malicious and selfish behavior node is to disrupt communication and conserve own resources, respectively. Resource conservation by selfish nodes would also ultimately degrade the overall network performance. Simulations result showed an enormous decrease in packet delivery ratio and extensive packet dropping by these malicious and misbehaving nodes.

An improved mobile ad hoc network ondemand routing protocol which is based on AODV by controlling the broadcasting of RREQ information is proposed. This protocol [24] analysis the lifetime of node when implementing routing discovery, and avoiding the unnecessary information sending efficiently. By comparing AODV with IAODV in the same scenario, the new protocol is much better than AODV in terms of packet delivery ratio as well as routing load. An energy consumption model [25] to calculate the energy-factor of the nodes is considered and then a trust based protocol for energyefficient routing is proposed. A trust module to track the value of routing metric is adopted. Simulation results show that the proposed protocol reduces delay, routing overhead, and increases packet delivery ratio by consuming less energy compared to AODV and DSR. 
Continuous evaluation of node's performance and collection of neighbor node's opinion value about the node are used to calculate the trust relationship of this node with other nodes. A perfect trust model [26] is introduced in the network layer to establish secure route between source and destination without any intruders or malicious nodes. Thus the existing AODV routing protocol has been modified in order to adapt the trust based communication feature. Proposed trust based routing protocol equally concentrates both in node trust and route trust. A routing algorithm [27] which adds a field in request packet which stores trust value indicating node trust on neighbor is proposed. Based on level of trust factor, the routing information will be transmitted depending upon highest trust value among all. This not only saves the node's power by avoiding unnecessary transmitting control information but also in terms of bandwidth (channel utilization), which is very important in case of MANET. The malicious node can attack on the control packet and misbehave in the network. The malicious node, which may or may not be trusted node. Here, a trusted path irrespective of shortest or longest path which can be used for communication in the network is proposed. Route trust value is calculated on the complete reply path which can be utilized by source node for next forthcoming communication in the network.

A security-enhanced AODV routing protocol [28] called R-AODV (Reliant Ad hoc On-demand Distance Vector Routing) is presented. The implementation is done by a modified trust mechanism known as direct and recommendations trust model and then incorporating it inside AODV which will allow AODV to not just find the shortest path, but instead to find a short path that can be trusted. This enhances security by ensuring that data does not go through malicious nodes that have been known to misbehave. The R-AODV protocol does provide a more reliable data transfer compared to the normal AODV if there are malicious nodes in the MANET.

Two kinds of approaches [29] are applied to a wellknown routing protocol called SAODV in order to improve its performance and to offer more resilience to attack from malicious nodes authenticated by the network. A preventive approach based on a cryptographic mechanism and a reactive approach to detect the anomalous and malicious behavior of nodes is considered. An extension of SAODV to offer Intrusion Detection mechanism (IDM) and trustbased mechanism (TBM) to promote the collaboration of the cooperating node and penalize the selfish nodes are proposed. The extended and proposed protocol SAODV-SDO is presented and simulation results performed in order to show the effectiveness of the proposed protocol in comparison with AODV and SAODV.

\section{PROPOSED WORK}

In Mobile ad hoc network routing is affected due to the dynamic nature of nodes. In spite of this dynamic topology nodes communicate with each other and exchange data on the network. The architecture of the proposed work is shown in Fig. 1. Trust based work [30] designed \& implemented and for every run it takes into account the nodes which have good success rate and failure rate. Trust is calculated based on success and failure rate and trust values for nodes are stored

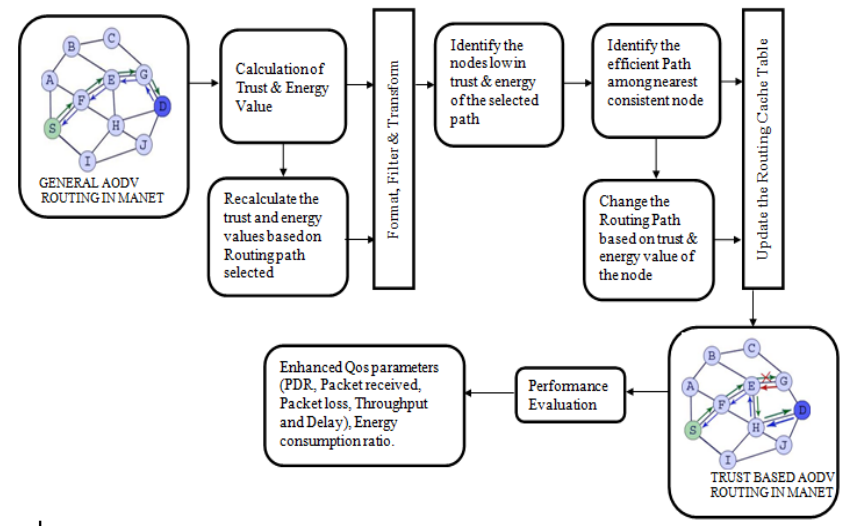

Fig. 1. Architecture of the proposed work

TABLE I

TRUST VALUE CALCULATION PARAMETERS

\begin{tabular}{llll}
\hline \hline Count Type & RREQ & RREP & Data \\
\hline Success & Qrs & Qps & Qds \\
Failure & Qrf & Qpf & Qdf \\
& & & \\
\hline \hline
\end{tabular}

separately for each node during simulation. When network starts, the initial trust value is 1 for all nodes. This value either increases or decreases based on nodes success or failure rate. All nodes are trusted nodes initially but trust value changes for every run of simulation. During simulation communication messages (RREQ and RREP) are exchanged between nodes in the network. RREP contains energy values also.

The trust level value calculation is based on the parameters shown in the Table I. The count field describes about two criteria, success rate (packets delivered successfully) and failure rate (packets lost or not delivered). RREQ and RREP are the route request and route reply respectively which are exchanged between nodes in the network. Data refers to the payload transmitted by the node in the routing path.

The parameter Qrs is defined as the query request success rate which is calculated based on number of neighboring nodes who have successfully received (RREQ) from the source node which has broadcasted it, Qrf defined as the query request failure rate which is calculated based on number of neighboring nodes which have not received the query request, Qps is defined as the query reply success rate which is calculated as successful replies (RREP) received by the source node which has sent the RREQ and Qpf is defined as the query reply failure rate which is calculated based on the number of replies not received by the source node for which RREQ was sent. Qds is defined as the data success rate calculated based on successfully transmitted data and Qdf is defined as data failure rate calculated based on data which have failed to reach destination.

\section{A. Trust calculation Pseudocode}

Step 1: Begin; For nodes i to $n$ do

Step 2: Initialize qrs, qps,qds //Success rate parameters Step 3: Initialize qrf, qpf, qdf // Failure rate parameters Step 4: Initialize QR, QP, QD // Intermediate values of request, reply and date respectively. 
Step 5: Calculate QR: $Q R=\sum_{i}^{n} \frac{q r s-q r f}{q r s+q r f}$

Step 6: Calculate QP: $\quad Q P=\sum_{i}^{n} \frac{q p s-q p f}{q p s+q p f}$

Step 7: Calculate QD: $Q D=\sum_{i}^{n} \frac{q d s-q d f}{q d s+q d f}$

Step 8: Calculate Trust Level Value:

$T L=T(R R E Q) * Q R+T(R R E P) * Q P+T(D A T A) * Q D$

// $\mathrm{T}$ is the time factorial of RREQ, RREP, DATA sent respectively

Step 9: For all neighbor nodes $\mathrm{j}$ to $\mathrm{n}$ do

Step 10 : Calculate Trust-Threshold: $T T=\sum_{i}^{n} \frac{T L}{n}$

Step 11: End

\section{B. Energy calculation procedure:}

In MANET, nodes energy also plays a key role. Node is selected for routing only if its energy level is also greater than the threshold value (average of energy values of the nodes). During simulation scenario energy values are displayed on top of each node. For every transmission the transmission power and reception power gets subtracted from its initial value of 100 Joules (initialized during simulation). Energy calculation is based on nodes sending and receiving rate.

Step 1: Do For all intermediate nodes which receive route request from a source node.

Step 2: Introduce energy for all nodes and set initial parameters values as initialenergy $=100$, maxenergy $=100$, nodes $=50$ and Nodeid ( unique id for each node)

Step 3: Calculate Intermedenergy based on event , time where events can be (event = "Tm" || event= "Rm" || event ="Im" event="Om") // Transmit mode, Receive mode, Idle mode and Overhear mode.

Step 4: Compute consumed energy for each node;

for (i in Intermednergy) \{

consumedenergy[i]=initialenergy-Intermedenergy[i]

totalenergy $+=$ consumedenergy[i]

if(maxenergy<consumedenergy[i]) \{

maxenergy $=$ consumedenergy[i]

nodeid=i $\}$ \}

Step 5: Node which sends route request collects energy value of all nodes which receive the request from source and reply to source along with their energy value (consumed energy).

Step 6: Compute threshold as total energy of nodes replied to node that has sent request by number of nodes replied.

Step 6. a:Calculate Energy-Threshold $E T=\sum_{i}^{n} \frac{\text { ConsumedEnergy }}{n}$

Step 7: Next hop node selected based on its energy value which is higher than the threshold.

Step 8: Compute average energy:

Averagenergy $=$ totalenergy/nodes

Step 9: End

Therefore the next hop node is selected based on the trust and energy value. To select the next hop node the trust and
TABLE II

COMPARISON OF PDR

\begin{tabular}{cccc}
\hline \hline & \multicolumn{3}{c}{ PDR(\%) } \\
Node Size & \multicolumn{3}{c}{ COMPARISON OF PDR } \\
\cline { 2 - 4 } & AODV & DSR & $\begin{array}{c}\text { Proposed } \\
\text { AODV }\end{array}$ \\
\hline 25 & 29.99 & 31.89 & 35.35 \\
50 & 31.50 & 44.64 & 74.47 \\
100 & 56.64 & 54.23 & 80.25 \\
\hline \hline
\end{tabular}

TABLE III

COMPARISON OF THROUGHPUT

\begin{tabular}{|c|c|c|c|}
\hline \multirow{2}{*}{ Node Size } & \multicolumn{3}{|c|}{ Throughput (bits/sec.) } \\
\hline & AODV & DSR & $\begin{array}{c}\text { Proposed } \\
\text { AODV }\end{array}$ \\
\hline 25 & 40 & 44 & 64 \\
\hline 50 & 130 & 299 & 367 \\
\hline 100 & 3175 & 873 & 8276 \\
\hline
\end{tabular}

TABLE IV

COMPARISON OF DELAY

\begin{tabular}{cccc}
\hline \hline & \multicolumn{3}{c}{ Delay (sec.) } \\
\cline { 2 - 4 } Node Size & AODV & DSR & $\begin{array}{c}\text { Proposed } \\
\text { AODV }\end{array}$ \\
\hline 25 & 0.235 & 0.375 & 0.081 \\
50 & 1.415 & 2.423 & 0.806 \\
100 & 6.799 & 9.292 & 3.516 \\
\hline \hline
\end{tabular}

energy value of all neighboring nodes from current source node is calculated and finally a node which has highest value than the threshold is selected as next hop node for the current routing. For example, Route starts from node N1 and next hop node N2 is selected. Now to select next hop node for N2 its neighbors are identified and their trust values and energy values are calculated. If $\mathrm{N} 3, \mathrm{~N} 4, \mathrm{~N} 5, \mathrm{~N} 6, \mathrm{~N} 7$ are the neighboring nodes of $\mathrm{N} 2$ then trust and energy value for all

these nodes are collected and an average of this is identified and this value is set as threshold value for selecting the next hop node for N2 only. The node which has the highest trust and energy value than the threshold will be selected as next hop node. Threshold value is calculated dynamically for every next hop node selection in each run. The nodes which are not selected for the current transmission based on their trust and energy value cannot be tagged as unfit node and can serve as best trusted node for another transmission based on the scenario. Thus the consideration of node's trust value and its energy value makes the routing more efficient compared with traditional AODV and DSR.

\section{RESULTS}

The performance of proposed AODV protocol is analyzed using NS-2 simulator. The network is designed using network simulator with 25, 50 and 100 nodes. General AODV \& DSR are simulated initially and its Qos metrics are observed. Proposed AODV is simulated and its results are also 


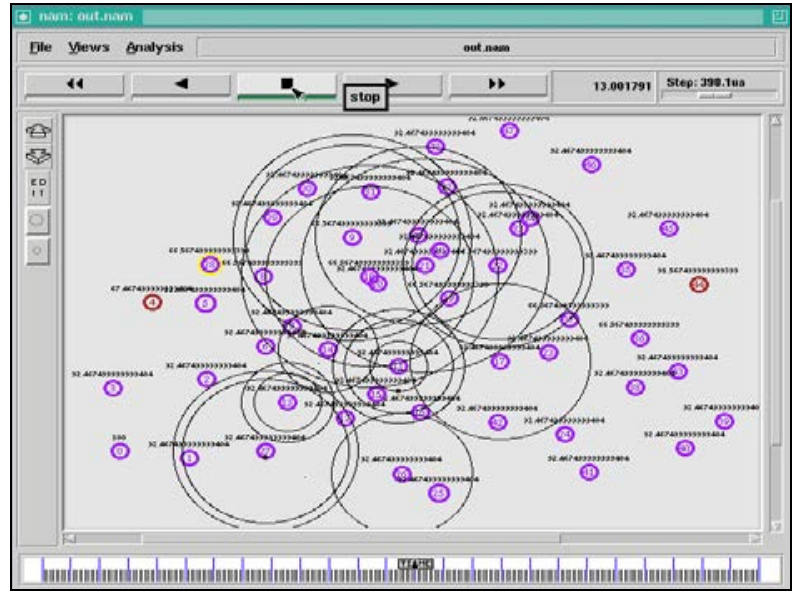

Fig. 2. Snapshot showing source and destination.

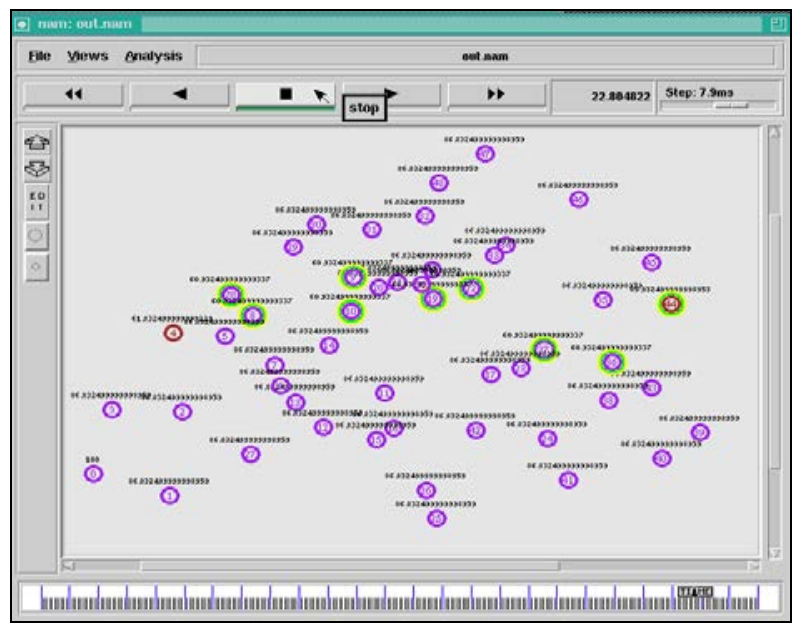

Fig. 3. Snapshot showing the misbehaving nodes.

observed. Results are compared in terms of Packet delivery ratio, Throughput, Delay and Energy consumption ratio. The proposed AODV shows good improvement in Qos metrics. PDR and Throughput are higher, delay is reduced and energy consumption is also less compared with general AODV and DSR as shown in Table II, Table III and Table IV.

Initially when trust model alone was incorporated the PDR was increased by $15 \%$. Now the trust and energy schemes are combined together and implemented in the proposed AODV. On an average of $20 \%$ increase is shown in PDR. This increase is because nodes are selected based on their trust and energy value. The next hop node selected to complete the path form source to destination, is based on its trust and energy value. This trust and energy based routing assures an efficient routing and avoids misbehaving nodes in routing path. The proposed AODV has also shown a decreased delay and an increase in throughput. Nodes which are trusted and with high energy levels are only used for routing path which ultimately reduces the time taken to reach the destination. Energy is vital for a node to perform in the network. Nodes with good trust may be selected for routing but inefficient energy may lead to poor performance of the node towards routing. This scheme selects node for only routing which has good energy values. The energy consumption of nodes are reduced so that they maintain good energy levels for future transmission and extend their lifetime in the network. Compared with proposed AODV, DSR and traditional AODV have consumed more energy.

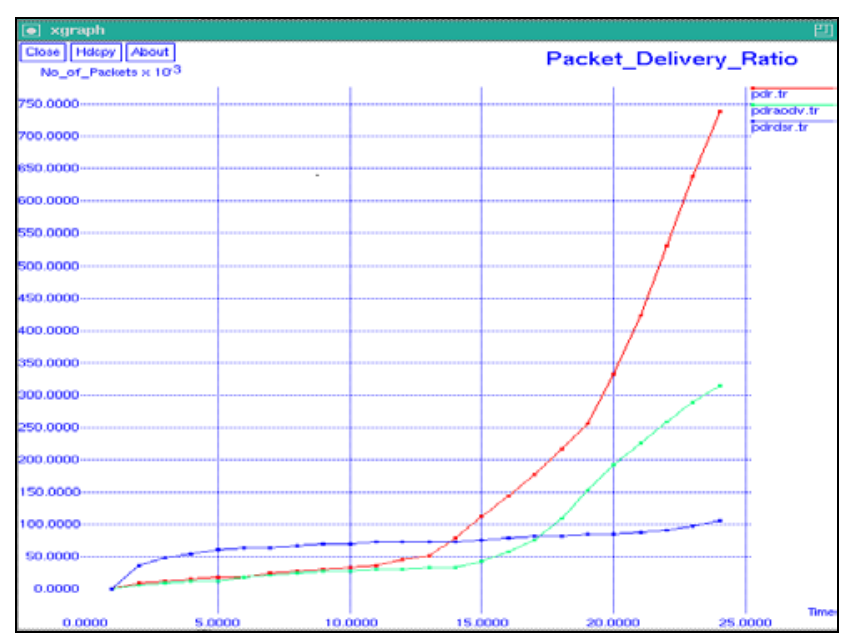

Fig. 4. Comparison of general AODV, DSR and proposed AODV PDR

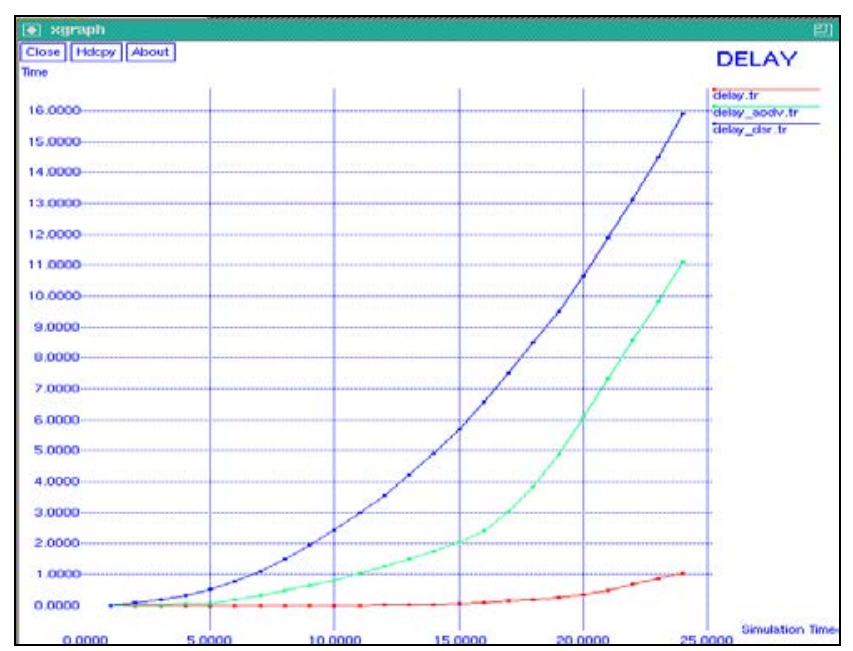

Fig. 5. Comparison of general AODV, DSR and proposed AODV Delay

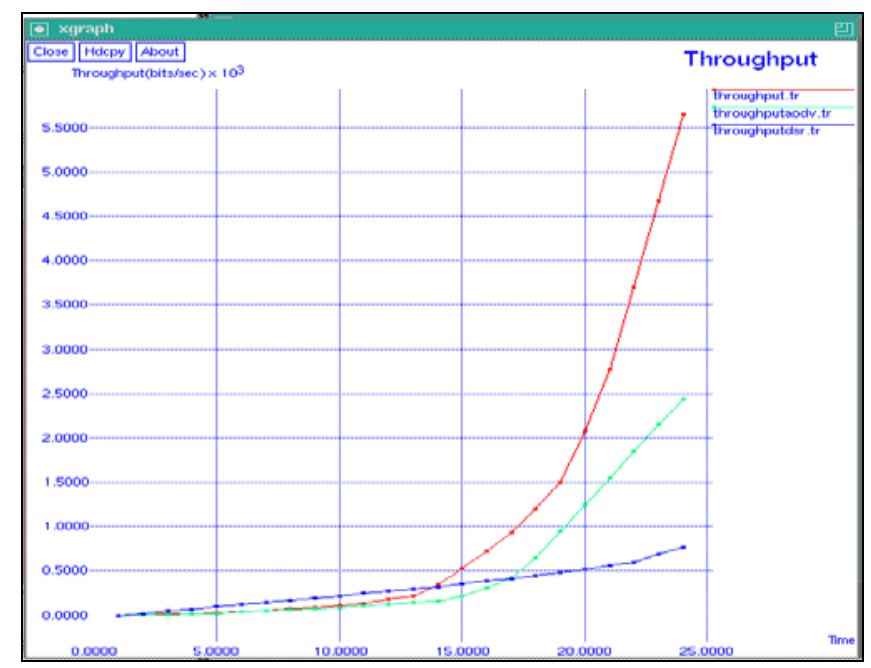

Fig. 6. Comparison of general AODV, DSR and proposed AODV Throughput 


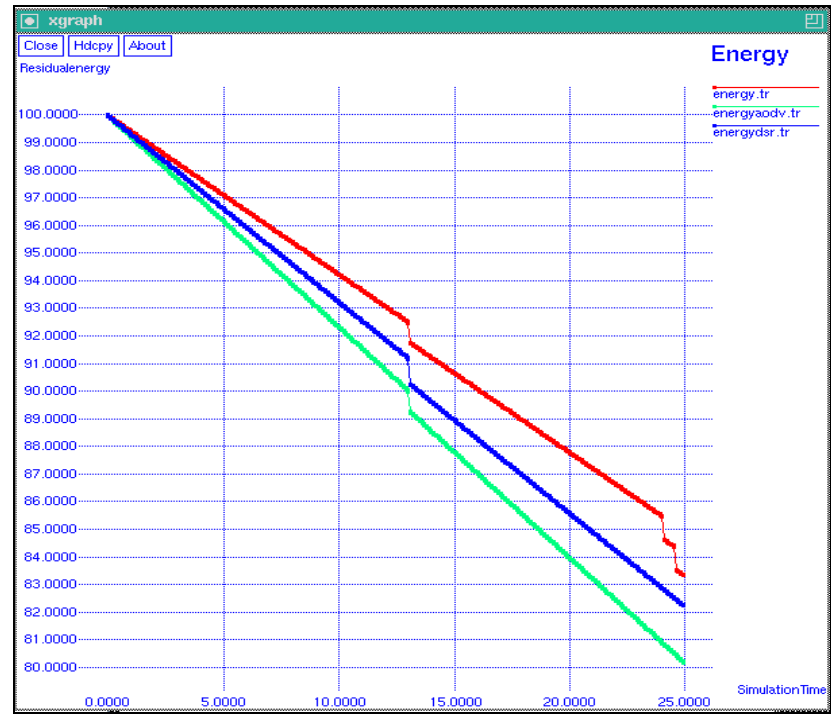

Fig. 7. Comparison of general AODV, DSR and proposed AODV energy consumption

\section{CONCLUSION}

Nodes in MANET may misbehave or drop nodes during routing which affects the Qos parameters and brings down the performance of the Network. A trust model is proposed which identifies misbehaving nodes the routing path and isolates those nodes from routing and selects an alternate path for efficient routing and also improves the QOs performance. The trust factor is calculated based on the nodes success rate and failure rate of transmission. Though node is trusted if it has not got enough energy in it becomes ineffective for routing. Therefore Energy is also considered for routing where in node should have sufficient energy for taking part in routing. Simulation results show good improvement in Qos metrics. The results of the proposed AODV protocol are compared with traditional AODV and DSR protocol. In proposed AODV protocol, Packet delivery ration is increased, throughput is increased and Delay is reduced. Energy consumption is reduced in proposed protocol. The work can be used in scenarios where transmission needs to be really secured and reliable like any sort of emergency situations, Military fields, etc. The Proposed protocol can be compared with many other Reactive and Proactive protocols and virtual energy concepts can be introduced in future works.

\section{REFERENCES}

[1] Remondo, “Tutorial on wireless ad hoc networks", in proc.Second International Conference in Performance Modeling and Evaluation of heterogeneous networks, 2004. pp. 216-220.

[2] C.E.Perkins and E.Royer, "Ad-hoc on-demand distance vector routing", in proc. Second IEEE Workshop on Mobile Computing Systems and Applications, LA, USA, 1999, pp. 90100.

[3] D.B.Johnson and D.A.Maltz, "Dynamic Source Routing in Ad Hoc Wireless Networks,” Kluwer, 1996, pp. 246-258.

[4] V.Park and M.S.Corson, "A highly adaptive distributed routing Algorithm for mobile wireless networks", in proc. IEEE INFOCOM, Kobe, Japan, 1997, pp. 1405-1413.

[5] C.K.Toh, "A novel distributed routing protocol to support adhoc mobile computing", in proc. fifteenth IEEE Annual
International Phoenix Conference on Computers and Communications, 1996, pp. 480-486.

[6] R.Dube, C.D.Rais, K.Y.Wang and S.K.Tripathi. (1997, may). On Signal stability-based adaptive routing (SSA) for ad hoc mobile networks. IEEE Personal Communications. [online]. 4(1)), pp. 36-45. Available: http://pdos.csail.mit.edu/decouto/papers/dube97.pdf.

[7] G.Aggelou and R.Tafazolli, "RDMAR: a bandwidth-efficient routing protocol for mobile ad hoc networks", in proc. WoWMoM, 1999, pp. 26-33.

[8] C.E.Perkins and P.Bhagwat, "Highly dynamic destination sequenced distance-vector routing (DSDV) for mobile computers", in proc. ACM Special Interest Group on Data Communication, London, UK, 1994, pp. 234-244.

[9] S. Murthy and J.J.Garcia-Luna-Aceves, "A routing protocol for packet radio networks", in proc. ACM First International Conference on Mobile Computing and Networking, CA, USA, 1995, pp. 86-95.

[10] S. Murthy and J.J.Garcia-Luna-Aceves. (1996, aug). On An efficient routing protocol for wireless networks. ACM Mobile Networks and Applications, Special Issue on Routing in Mobile Communication Networks.[online]. 1(2), pp. 183-197. Available: http://link.springer.com/article/10.1007\%2FBF01193336

[11] G.Pei, M.Gerla and T.W.Chen, "Fisheye state routing: a routing scheme for ad hoc wireless networks", in proc. ICC, LA, 2000, pp. 70-74.

[12] I.Jawhar and J. Wu, "Quality of Service Routing in Mobile Ad Hoc Networks,” in M Cardei, I Cardei \& DZ Du (eds), Resource Management and Wireless Networking, Kluwer Academic Publishers. 1999, pp 152-168.

[13] T.Beth, M.Borcherding and B.Klein, "Valuation of trust in open networks", in proc. ESORICS, 1994.

[14] K. S. Cook, "Trust in Society," in Russell Sage Foundation Series on Trust, New York. vol. 2, 2003, pp. 22-30

[15] K.Muthumayil, V.Rajamani and S.Manikandan, “A novel cross layered energy based ad hoc on-demand routing protocol for MANETs”, in proc. ICoAC, 2011, pp. 276-281.

[16] Arash Dana, Golnoosh Ghalavand, Azadeh Ghalavand and Fardad Farokhi,. (2011, May). On A Reliable routing algorithm for Mobile Adhoc Networks based on fuzzy logic. International Journal of Computer Science Issues.[online]. 8(3), pp. 128 133. Available: http://ijcsi.org/papers/IJCSI-8-3-1-128-133.pdf.

[17] Yunhuai Liu, Yanmin Zhu, Lionel M. Ni and Guangtao Xue. (2011, Dec). On A Reliability-oriented transmission service in wireless sensor networks. IEEE Transactions on Parallel and Distributed Systems.[online]. 22(12), pp. 2100 - 2107. Available: ieeexplore.ieee.org > ... > Parallel and Distributed Syst

[18] MouZonghua and Meng Xiaojing, "A modified AODV routing protocol based on route stability in MANET", in proc. 4th IET International Conference on Wireless, Mobile \& Multimedia Networks, 2011, pp. 63 - 67.

[19] A.Bagwari, R.Jee, P. Joshi and S.Bisht, "Performance of AODV Routing Protocol with Increasing the MANET Nodes and Its Effects on QoS of Mobile Ad Hoc Networks”, in proc. International Conference on Communication Systems and Network Technologies, 2012 , pp. 320-324.

[20] A.Akhter and T.Sanguankotchakorn, " Modified AODV for multi-constrained QoS routing and performance optimization in MANET”, in proc. International Conference on Electrical Engineering/Electronics Computer Telecommunications and Information Technology, 2010 , pp. $234-238$.

[21] Chee-Wah Tan and S.K.Bose, "Investigating Power Aware AODV for Efficient Power Routing in MANETs", in proc. Fifth International Conference on Information, Communications and Signal Processing, 2005, pp. $584-588$. 
[22] Jin-ManKim and Jong-WookJang, "AODV based Energy Efficient Routing Protocol for Maximum Lifetime in MANET”, in proc. International Conference on Internet and Web Applications and Services, 2006, pp. 77.

[23] M.S.Alkatheiri, JianweiLiu and A.R.Sangi, “AODV routing protocol under several routing attacks in MANETs", in proc. ICCT, 2011, pp. $614-618$.

[24] Zhang Jianwu, ZouJingyuan and ZhaoQi, " MANET Routing Protocol for Improving Routing Discovery Based on AODV”, in proc. International Conference on Networks Security, Wireless Communications and Trusted Computing, 2009, pp.197 - 200.

[25] S.Sarkar and R.Datta, “A trust based protocol for energyefficient routing in self-organized MANETs", in INDICON, 2012, pp. $1084-1089$.

[26] A.M.Pushpa, "Trust based secure routing in AODV routing protocol", in proc. IMSAA, 2009, pp.1-6.

[27] R.S.Mangrulkar and M.Atique, "Trust based secured adhoc On demand Distance VectorRouting protocol for mobile adhoc network “,in proc. WCSN, 2010, pp.1-4.

[28] H.S.Jassim, S.Yussof, Tiong Sieh Kiong, S.P.Koh and R.A.Ismail, "A routing protocol based on trusted and shortest path selection for mobile ad hoc network”, in proc. MICC, 2009, pp.547 - 554.

[29] F.De Rango, "Improving SAODV protocol with trust levels management, IDM and incentive cooperation in MANET", in Wireless Telecommunications Symposium, 2009, pp. 1 - 8 .

[30] S.Sridhar and R.Baskaran, "Quality of service routing in MANET using trusted AODV (TS-AODV)", in proc. ICCCMIT, Chennai, 2014, pp. 58.

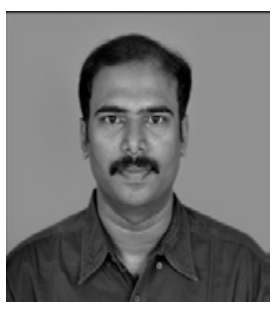

S. Sridhar received UG degree, B.Sc. Computer Science from the University of Madras, Chennai, India, in 1998, PG degree, Master of Computer Applications (MCA) from University of Madras, Chennai, India, in 2001 and another PG degree Master of Philosophy ( M.Phil.) in Computer Science from Periyar University, Salem, Tamil Nadu, India, in 2007. Currently pursuing the Ph.D. degree in computer science at Barathiyar University, Coimbatore, Tamil Nadu, India. Since 2001 he has been working with Department of Computer Applications (MCA), S.A.Engineering College, Chennia, Tamilnadu and currently heading the department. He has got more than 15 international publications. His research interests include Mobile Adhoc networks, Wireless sensor networks and Adhoc networks. He is an editorial member of 2 International Journals and reviewed many technical papers for conferences and journals.

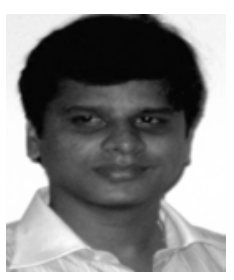

R. Baskaran obtained M.E. and Ph.D. in the field of Computer Science and Engineering in Anna University at Chennai, India. He is working as Associate professor in Department of computer science, Anna University, Chennai. He is having around a decade of experience as an academician and his research areas include Multimedia and principles, Software quality engineering, Software Agents and Distributed networking.

He has published around 75 research papers in National and International Journals and Conferences. He is a member of various forums. He is the editor and a reviewer of various journals. He is guiding research scholars working in area of software standards for Attributes Specific SDLC Models \& Evaluation and Metric Based Efficient Traffic Management. 